\title{
Perfil sociodemográfico y patrones de consumo de drogas de pacientes de cocaína y crack*
}

\section{Socio Demographic Profile and Usage Patterns of Crack and Cocaine Consumers}

\author{
Roberto Oropeza Tena* \\ Jennifer Lira Mandujano \\ María Rosales García \\ Universidad Michoacana de \\ San Nicolás de Hidalgo, México
}

Recibido: 2 de abril de 2013 Revisado: 15 de mayo de 2013 Aceptado: 25 de junio de 2013

\section{Resumen}

El objetivo de esta investigación fue conocer el perfil sociodemográfico y el patrón de uso de cocaína y crack, y su asociación con tabaco y otras sustancias entre usuarios que asisten a un tratamiento ambulatorio. Participaron 139 usuarios $(87,3 \%$ hombres y $12,7 \%$ mujeres), que hicieron parte del 'Tratamiento breve para usuarios de cocaína' (TUBC), de la Facultad de Psicología de la Universidad Nacional Autónoma de México (UNAM). Los instrumentos utilizados fueron el cuestionario de preselección y la entrevista inicial. Encontramos que hay diferencias en el perfil de los consumidores de coca y los de crack. Sigue siendo mayor la proporción de consumidores de coca que de crack; los consumidores de coca tienen mayor escolaridad que los consumidores de crack; la mayoría de ambos consumidores han conservado un empleo. Más usuarios de coca habían asistido a un tratamiento. Estos resultados son muy útiles para ampliar el conocimiento sobre las características que tienen este tipo de consumidores y ayudar a perfilar los tratamientos específicos que cada uno requiere.

Palabras clave: cocaína, tabaco, drogas, perfil sociodemográfico.

* Artículo de investigación.

Correspondencia: Roberto Oropeza Tena, Profesor investigador, Posgrado de Psicología, Universidad Michoacana de San Nicolás de Hidalgo. Dirección Av. Solidaridad 734, Col. Félix Ireta, CP 58070, Morelia, Michoacán. Correo electrónico: scherzo2112@yahoo.com 


\begin{abstract}
The aim of this investigation was to recognize the socio demographic profile of crack and cocaine consumers that have attended an ambulatory treatment. As a well as, to define the crack and cocaine usage patterns and their association with tobacco and other substances amongst these groups of consumers. 39 users took part in the investigation (87.3\% men and 12.7 women) said participants had all received the Brief treatment for cocaine users (TUBC) at the faculty of psychology of the UNAM. The items used in this study were: a preselecting questionnaire and an intake interview. Our results showed that there are profile differences amongst cocaine and crack consumers. Firstly, the percentage of cocaine consumers is still higher than the percentage of those who take crack. Secondly, Cocaine consumers have a higher grade of education than crack consumers. Thirdly, there were more cocaine users that had previously attended a treatment. However, the majority of subjects in both groups had managed to maintain their Jobs. These results are very useful to widen the knowledge about the characteristics of each type of consumer; what is more, they help us define the specific treatment each type of consumer requires.
\end{abstract}

Keywords: cocaine, tobacco, drugs, socio demographic profile.

El consumo de drogas es un problema que afecta a todas las culturas. Durante los siglos XX y XXI ha aumentado considerablemente su consumo en prácticamente todo el mundo. Entre las drogas que más se han extendido está la cocaína, y México ha sido especialmente vulnerable a este aumento. En la primera Encuesta Nacional de Adicciones (ENA) (Secretaría de Salud, 1988) practicada en México, el consumo de esta sustancia alguna vez en la vida en la población general ocupaba el tercer lugar entre las drogas ilegales $(0,33 \%)$. A partir de la tercera ENA (Secretaría de Salud, 1998) subió al segundo lugar (1,45\%); en la Quinta Encuesta Nacional de Adicciones (Secretaría de Salud, 2008) la cocaína se sigue manteniendo en ese puesto (2,37\%). La Sexta Encuesta Nacional de Adicciones (Secretaría de Salud, 2011) indica que, en el último año, la cocaína sigue siendo la segunda droga ilegal más consumida en México. También hubo un aumento del consumo de crack, aunque solo la Quinta Encuesta Nacional de Adicciones informa su consumo alguna vez en la vida en la población general (0,59\%). En México, así como en otros países, el consumo de cocaína está muy por debajo del consumo de alcohol, tabaco y cannabis, drogas más tradicionales (Cañuelo y García, 2002; Secretaría de Salud, 2008).

La cocaína es un poderoso estimulante, que da a los consumidores una sensación de confianza, vigor, euforia y de reducción del apetito. Aumenta la capacidad física, reduce el cansancio y hace sentir fuerte al usuario. El uso de esta droga se prohibió a principios del siglo XX y su consumo fue declinando poco a poco, para aumentar nuevamente a partir de 1970. Por otro lado, el brote del crack fue en 1985, en las Bahamas; así se inició una nueva fase en la historia de la cocaína, respecto a su grado de toxicidad (Ferreira y Martini, 2001). Además de todos los problemas cognitivos (Verdejo-García y Pérez-García, 2007), esta forma de cocaína tiene efectos más rápidos e intensos, pero de menor duración, por lo que tiene mayor poder adictivo, al agotar la condición física, psíquica y financiera de los consumidores, que muchas veces se envuelven en actividades ilícitas para mantener su adicción. Así mismo, su consumo impacta negativamente en el rendimiento escolar (Correia, Torres, Maia, de Sousa y Santos, 2010).

En este sentido, Ferreira, Turchi, Laranjeira y Castelo, (2003), a partir de un estudio efectuado sobre el perfil sociodemográfico de personas dependientes de cocaína internados en hospitales psiquiátricos de la región metropolitana de Sao Paolo, concluyen que los usuarios de crack están más expuestos a situaciones de violencia, lo que sugiere mayor vulnerabilidad y aumento de factores de riesgo para la salud de esa población. 
Por eso, Ribeiro, Dunn, Sesso, Dias \& Laranjeira (2006) afirman que los consumidores de crack presentan mayor riesgo de muerte que la población general, teniendo como una de sus principales causas los homicidios. Además, sugieren que futuros estudios deben analizar los datos sociodemográficos de usuarios de esta sustancia, considerando su historia de consumo de drogas, así como las influencias culturales y económicas, para poder determinar pronósticos futuros y construir nuevas estrategias para abordar este grave problema social. De la misma forma, se percibe que el abuso o dependencia de otras sustancias psicoactivas es común en los consumidores de crack y cocaína.

En Brasil se ha instrumentado una serie de estudios sobre el perfil de consumidores de cocaína y crack que asisten a servicios de salud (Duailibi, Ribeiro y Laranjeira, 2008; Ferreira et al., 2003; Guimarães, Vieira dos Santos, Cavalari de Freitas y Araujo, 2008).

Guimarães et al., (2008) llevaron a cabo un estudio en Brasil con 30 hombres consumidores de crack en una unidad de desintoxicación de un hospital psiquiátrico, con el objetivo de identificar el perfil sociodemográfico y de consumo de sustancias psicoactivas. Encontraron que estos consumidores son principalmente adultos jóvenes, de raza blanca, con un promedio de edad de 27,3 años, en situación de subempleo o desempleo. Hallaron también que estos consumidores presentan problemas de ansiedad y depresión.

Por su parte, a Herrero, Domingo-Salvany, Torrens, Brugal y Gutiérrez (2008) también les interesó conocer el perfil de consumidores de cocaína. Participaron 120 consumidores de esta sustancia (38,8\% mujeres), con un promedio de edad de 23,8 años, el $85 \%$ solteros que no asistían a tratamiento; el 46,6\% tenían al menos el bachillerato. El $96,7 \%$ de los participantes tenían una educación posterior a primaria, casi el $50 \%$ era empleado, un tercio vivía con sus parientes y el $21 \%$ tenía algún antecedente criminal. La principal ruta de consumo (91\%) era esnifada. En promedio, usaron 1,82 sustancias adictivas.

Sánchez y Nappo (2002) querían conocer la secuencia de drogas consumidas por usuarios de cocaína y/o crack, y encontraron que inician con tabaco y/o alcohol, seguidas de marihuana, cocaína y crack, en la mayoría de los consumidores estudiados. Cañuelo y García (2002) mencionan que es muy común el consumo de la cocaína como sustancia estimulante, para contrarrestar los efectos depresivos y sedativos del alcohol, teniendo como efecto un estado de vigilia y actividad más prolongado. El problema del consumo de ambas sustancias es que produce un nuevo metabolito, el cocaetileno, que puede tener efectos muy nocivos en la salud del consumidor. Entre otros problemas, incrementa la producción de metabolitos tóxicos, poniendo en serio riesgo el hígado (Ladero y Martín, 2009).

Este tipo de estudios transversales son importantes para observar las características de los consumidores y desarrollar tratamientos específicos para las necesidades y problemáticas de estos pacientes. Por otro lado, es necesario profundizar en el conocimiento de esta población, con el fin de contribuir de forma eficaz en el aumento de las tasas de abstinencia de estas drogas. Por lo tanto, el objetivo de esta investigación fue conocer el perfil sociodemográfico y el patrón de uso de cocaína y crack, y su asociación con tabaco y otras sustancias entre usuarios que asisten a un tratamiento ambulatorio. Es un estudio descriptivo y transversal.

\section{Método}

\section{Participantes}

En esta investigación participaron 139 usuarios (87,3\% hombres y $12,7 \%$ mujeres) que hicieron al menos la entrevista de admisión del 'Tratamiento breve para usuarios de cocaína' (TBUC, Oropeza, 2003), que se otorga en el Centro de Prevención y Atención en Adicciones Héctor E. Ayala Velázquez (CEPREAA), de la Facultad de Psicología de la Universidad Nacional Autónoma de México (UNAM). Todos eran consumidores de crack y/o cocaína. En promedio, tenían una edad de $\bar{x}=28,44$ años (DE $7,38)$, con un rango de 17 a 52 años.

Los criterios de inclusión para participar en el estudio era que: a. los usuarios fueran mayores de 
18 años, b. la droga de impacto fuera cocaína o crack, y c. aceptaran participar en la investigación, con la firma un consentimiento informado.

\section{Instrumentos}

- Cuestionario de preselección (Oropeza, 2003). Consta de 4 reactivos dicotómicos y se usa para ver si el usuario cumple con los criterios de preselección.

- Entrevista inicial (Oropeza, 2003). Consta de 71 reactivos con diferentes opciones de respuesta (dicotómicas, opciones, tipo Likert o abiertas). Tiene 3 secciones: a. ficha de identificación (13 reactivos); b. historia del consumo de la cocaína y otras sustancias (44 reactivos); c. problemas asociados con el consumo de cocaína y otras sustancias (14 reactivos).

- Consentimiento informado (Oropeza, 2003), es un formato que firma el usuario (y el terapeuta), donde accede a que sus datos se usen en investigación y se le indica sobre la confidencialidad de la información dada.

\section{Procedimiento}

El TBUC es un modelo de tratamiento breve, de corte cognitivo conductual, dirigido a consumidores de cocaína. Consta de dos sesiones de evaluación, ocho tópicos de tratamiento y tres sesiones de seguimiento. Se otorga individualmente, es ambulatorio, las sesiones son semanales de 1,30 hora cada una. El TBUC ha demostrado ser un tratamiento útil para alcanzar la abstinencia de la cocaína y el crack (Oropeza, Medina-Mora y Sánchez-Sosa, 2007).

Los usuarios que llegaban al CEPREAA llenaron una solicitud de atención. Se les programó una primera sesión de evaluación donde se analizaban varios aspectos asociados con el consumo de cocaína y otras sustancias adictivas. En esta sesión también se revisaba que el usuario cumpliera con los criterios de inclusión. Si el paciente los cubría, se le explicaban las características generales del TBUC; se le indicaba que los datos obtenidos en los instrumentos de evaluación podían ser usados en investigación; y por último, se señalaba que su participación era voluntaria, además de que no habría datos que pudieran identificarlo. Si el usuario aceptaba participar, tanto él como el terapeuta firmaban el consentimiento informado; posteriormente, se le hacía la entrevista inicial. Los instrumentos fueron aplicados por psicólogos específicamente entrenados en el TBUC, en entrevistas personales individuales. Al momento de la entrevista, los participantes no podían estar intoxicados por el consumo de alguna droga.

Para esta investigación se revisaron los expedientes de 139 pacientes que habían participado en el TBUC. La muestra fue de sujetos voluntarios por conveniencia.

\section{Análisis estadísticos}

Para el análisis descriptivo de los datos se utilizó el SPSS 17. Los resultados de las variables sociodemográficas y de los patrones de uso de drogas fueron presentados por medio de promedios de las variables numéricas y por porcentajes para las variables categóricas. Para establecer las comparaciones de promedios entre dos grupos de variables de intervalo se utilizó la prueba T de Student, y para grupos de variables ordinales se utilizó la $U$ de Mann-Whitney. Para comparar variables categóricas entre dos grupos se utilizó la $\chi^{2}$. El nivel de significancia a se fijó en el $5 \%$.

\section{Resultados}

La muestra estudiada estuvo compuesta por 139 pacientes. Respecto al género, había más hombres consumidores de cocaína (46\%) y crack $(40,3 \%)$, que mujeres $(7,9 \%$ consumidoras de cocaína y $5,8 \%$ de crack). En relación con su estado civil, el 58,2\% eran solteros; el 37,3\%, con pareja estable, y el 4,4\%, divorciados. Tenían un rango de estudios de 6 a 17 años, con una $\bar{X}=11,58$ $(s=3,15)$. En ese sentido, los consumidores de cocaína tenían mayor escolaridad que los consumidores de crack. Se hizo una U de Mann-Whitney y se encontró que había una diferencia estadísticamente significativa entre los grupos, donde los 
consumidores de cocaína tenían una escolaridad más alta $(u=1902,[74,63, p<0,05])$ que los consumidores de crack. Por otro lado, la mayoría de ambos tipos de consumidores trabajaban (76,5\%). En la tabla 1 se presentan otros aspectos sociodemográficos de los participantes.

Tabla 1.

Aspectos sociodemográficos de los consumidores de coca y crack

\begin{tabular}{|c|c|c|c|c|c|}
\hline \multirow{2}{*}{\multicolumn{2}{|c|}{ Aspectos demográficos }} & \multicolumn{2}{|c|}{ Cocaína } & \multicolumn{2}{|c|}{ Crack } \\
\hline & & $N$ & $\%$ & $N$ & $\%$ \\
\hline \multirow{2}{*}{ Género } & Masculino & 64 & 46 & 56 & 40,3 \\
\hline & Femenino & 11 & 7,9 & 8 & 5,8 \\
\hline \multirow{5}{*}{ Escolaridad } & Primaria & 2 & 1,5 & 5 & 3,6 \\
\hline & Secundaria & 21 & 15,3 & 23 & 16,8 \\
\hline & Bachillerato & 22 & 16,1 & 19 & 13,9 \\
\hline & $\begin{array}{l}\text { Carrera } \\
\text { técnica }\end{array}$ & 4 & 2,9 & 3 & 2,2 \\
\hline & Profesional & 25 & 18,2 & 13 & 9,5 \\
\hline \multirow{3}{*}{$\begin{array}{l}\text { Situación } \\
\text { laboral }\end{array}$} & Trabaja & 50 & 42 & 41 & 34,5 \\
\hline & Estudia & 2 & 1,7 & 2 & 1,7 \\
\hline & $\begin{array}{l}\text { Ni trabaja } \\
\text { ni estudia }\end{array}$ & 13 & 10,9 & 11 & 9,2 \\
\hline
\end{tabular}

Para el 24\% de los consumidores de cocaína y el 21,6\% de los consumidores de crack este era su primer tratamiento de adicciones (tabla 2). En total, el $68 \%$ de ambas poblaciones ya había estado previamente en algún tratamiento para trabajar su problema con la cocaína o el crack.

Tabla 2.

Participación en tratamientos de drogas

\begin{tabular}{lrcccc}
\hline \multirow{2}{*}{ Internaciones } & \multicolumn{3}{c}{ Cocaína } & \multicolumn{2}{c}{ Crack } \\
\cline { 2 - 6 } & & $N$ & $\%$ & $N$ & $\%$ \\
\hline ¿Ha estado en & Sí & 38 & 30,4 & 30 & 24 \\
$\begin{array}{l}\text { otro tratamiento } \\
\text { de drogas? }\end{array}$ & No & 30 & 24 & 27 & 21,6 \\
\hline
\end{tabular}

Sobre la edad de inicio del consumo de cocaína, se encontró que eran bastante jóvenes cuando iniciaban, ya que en promedio lo hacían a los 21,46 años ( $s=6,89$ años). Respecto a la forma de presentación de la sustancia, el 53,5\% consumían cocaína y el 45,7\% consumían crack (tabla 3). En cuanto a los años de consumo, la mayoría llevaba de 0 a 5 años usando la sustancia. Se compararon ambos grupos con la $U$ de Mann-Whitney y no se encontraron diferencias estadísticamente significativas. En relación con la cantidad consumida por ocasión, había mayor consumo entre los usuarios de crack que los de cocaína. Se usó una prueba T para ver si había diferencias estadísticamente significativas entre ambos grupos, pero no se encontraron.

Acerca de los días de hospitalización en el último año por el consumo de cocaína, aunque los consumidores de crack estuvieron más tiempo hospitalizados que los de cocaína, no se encontraron diferencias estadísticamente significativas entre ambos grupos. En cuanto al tiempo invertido en el consumo, no se encontraron diferencias estadísticamente significativas. Tampoco se encontraron diferencias respecto a si consumía solo o acompañado.

Tabla 3.

Características de uso de cocaína

\begin{tabular}{|c|c|c|c|c|c|}
\hline \multirow{2}{*}{\multicolumn{2}{|c|}{ Variables }} & \multicolumn{2}{|c|}{ Cocaína } & \multicolumn{2}{|c|}{ Crack } \\
\hline & & $N$ & $\%$ & $N$ & $\%$ \\
\hline \multicolumn{2}{|c|}{$\begin{array}{c}\text { Forma de presentación } \\
\text { de consumo }\end{array}$} & 75 & 53,5 & 64 & 45,7 \\
\hline \multirow{4}{*}{ Años de consumo } & 0 a 5 & 37 & 30,3 & 32 & 26,2 \\
\hline & 6 a 10 & 23 & 18,9 & 19 & 15,6 \\
\hline & 11 a 15 & 5 & 4,1 & 4 & 3,3 \\
\hline & $>$ de 15 & 2 & 1,6 & 0 & 0 \\
\hline \multirow{4}{*}{$\begin{array}{l}\text { Cantidad } \\
\text { consumida por } \\
\text { ocasión (últimos } \\
90 \text { días) }\end{array}$} & 0 a $1 \mathrm{~g}$ & 26 & 24,07 & 17 & 15,74 \\
\hline & $>1$ a $3 \mathrm{~g}$ & 20 & 18,51 & 24 & 22,22 \\
\hline & $>3$ a $5 \mathrm{~g}$ & 6 & 5,55 & 7 & 6,48 \\
\hline & $>5 \mathrm{~g}$ & 5 & 4,62 & 5 & 4,62 \\
\hline $\begin{array}{l}\text { Días de } \\
\text { hospitalización } \\
\text { debido al consumo } \\
\text { (último año) }\end{array}$ & $\begin{array}{c}0 \\
1 \text { a } 5 \\
>\text { de } 5\end{array}$ & $\begin{array}{c}63 \\
4 \\
0\end{array}$ & $\begin{array}{c}58,8 \\
3,2 \\
0\end{array}$ & $\begin{array}{c}50 \\
4 \\
3\end{array}$ & $\begin{array}{c}40,3 \\
3,2 \\
2,4\end{array}$ \\
\hline \multirow{5}{*}{ Tiempo invertido } & $<1 \mathrm{~h}$ & 6 & 4,7 & 5 & 3,9 \\
\hline & $1 a<3 h$ & 13 & 10,1 & 7 & 5,4 \\
\hline & $3 a<7 h$ & 18 & 14 & 23 & 17,8 \\
\hline & $7 a<12 h$ & 13 & 10,1 & 13 & 10,1 \\
\hline & $>12 \mathrm{~h}$ & 18 & 14 & 13 & 10,1 \\
\hline \multirow{3}{*}{$\begin{array}{l}\text { ¿Con quién } \\
\text { consume? }\end{array}$} & Solo & 40 & 29,4 & 34 & 25 \\
\hline & Acompañado & 28 & 20,6 & 23 & 16,9 \\
\hline & Ambas & 5 & 3,7 & 6 & 4,4 \\
\hline
\end{tabular}


De la muestra total, nunca habían sido detenidos el $84,8 \%$ (tabla 4); lo cual es ligeramente mayor con el número de usuarios de crack, que de cocaína.

Tabla 4.

Número de arrestos asociados con el consumo de cocaína o crack

\begin{tabular}{ccccc}
\hline \multirow{2}{*}{ Arrestos } & \multicolumn{2}{c}{ Cocaína } & \multicolumn{2}{c}{ Crack } \\
\cline { 2 - 5 } & $\boldsymbol{N}$ & $\%$ & $\mathrm{~N}$ & $\%$ \\
\hline 0 & 58 & 46,4 & 48 & 38,4 \\
1 & 4 & 3,2 & 7 & 5.6 \\
2 & 2 & 1,6 & 1 & 0,8 \\
4 & 4 & 3,2 & 0 & 0 \\
6 & 0 & 0 & 1 & 0,8 \\
\hline
\end{tabular}

La tabla 5 revela algunos datos de uso concomitante con el uso de cocaína o crack, de consumo de drogas lícitas (alcohol y tabaco) e ilícitas (marihuana, pastillas e inhalantes). Respecto al consumo de alcohol, había más consumidores de alcohol entre los usuarios de cocaína que de crack. Se hizo una $\chi^{2}$ y se encontraron diferencias estadísticamente significativas entre ambas muestras $\left(\chi^{2}=4,864,1, p<0,027\right)$. Un uso aceptado de alcohol es hasta 4 tragos estándar por ocasión, y casi un $47 \%$ de los consumidores de cocaína y crack consumen más de esa cantidad por ocasión de consumo.

La Organización Panamericana de la Salud (OPS, 2000) indica que consumir más de cuatro copas por ocasión es ya un consumo de alto riesgo. No se encontraron diferencias estadísticamente significativas entre ambas muestras respecto a la cantidad de copas consumidas por ocasión. El consumo de tabaco también era frecuente. En general, los consumidores de crack consumían más cigarros diarios que los consumidores de cocaína; sin embargo, no se encontraron diferencias estadísticamente significativas entre ambos grupos.

Por otro lado, 56 personas de la muestra señalaron que consumen drogas ilegales. De la muestra total, una gran parte son consumidores de marihuana (51 personas); dos de inhalantes (thinner o diluyente), y tres de los consumidores de cocaína consumen anfetaminas (sustancia que no informan los consumidores de crack).
Tabla 5.

Asociación de uso concomitante de alcohol, tabaco y drogas ilícitas

\begin{tabular}{|c|c|c|c|c|c|c|}
\hline & \multirow{2}{*}{ Drogas } & & \multicolumn{2}{|c|}{ Cocaína } & \multicolumn{2}{|c|}{ Crack } \\
\hline & & & $N$ & $\%$ & $N$ & $\%$ \\
\hline \multirow{6}{*}{ Alcohol } & \multirow{2}{*}{$\begin{array}{l}\text { ¿Consume } \\
\text { alcohol? }\end{array}$} & Sí & 27 & & 13 & \\
\hline & & No & 31 & & 37 & \\
\hline & \multirow{4}{*}{$\begin{array}{l}\text { Tragos } \\
\text { estándar } \\
\text { por } \\
\text { ocasión de } \\
\text { consumo }\end{array}$} & 1 a 4 & 23 & 28,4 & 20 & 24,7 \\
\hline & & 5 a 8 & 14 & 17,3 & 12 & 14,8 \\
\hline & & 9 a 12 & 3 & 3,7 & 4 & 4,9 \\
\hline & & $>12$ & 2 & 2,5 & 3 & 3,7 \\
\hline \multirow{7}{*}{ Tabaco } & \multirow{2}{*}{$\begin{array}{l}\text { ¿Consume } \\
\text { tabaco? }\end{array}$} & Sí & 31 & & 18 & \\
\hline & & No & 27 & & 31 & \\
\hline & \multirow{5}{*}{$\begin{array}{l}\text { Número de } \\
\text { cigarrillos } \\
\text { diarios }\end{array}$} & 1 a 5 & 19 & 22,9 & 14 & 16,9 \\
\hline & & 6 a 10 & 9 & 10,8 & 11 & 13,3 \\
\hline & & 11 a 15 & 3 & 3,6 & 2 & 2,4 \\
\hline & & 16 a 20 & 13 & 15,7 & 5 & 6 \\
\hline & & Más de 20 & 4 & 4,8 & 3 & 3,6 \\
\hline \multirow{3}{*}{$\begin{array}{l}\text { Otras } \\
\text { drogas } \\
\text { ilegales }\end{array}$} & Marihuana & & 28 & 32,2 & 23 & 26,4 \\
\hline & Anfetaminas & & 3 & 3,4 & 0 & 0 \\
\hline & Inhalantes & & 1 & 1,1 & 1 & 1,1 \\
\hline
\end{tabular}

\section{Discusión}

Los consumidores que asistieron al tratamiento utilizaban la cocaína intranasalmente o el crack fumado, y prácticamente ninguno usaba cocaína por vía intravenosa. Respecto al consumo de cocaína y crack según la última ENA (SS, 2008) en cuanto al género en la población general, la proporción de hombres es de $4,1 \%$, mientras que el de mujeres es de 0,7\%. En nuestros datos tenemos que la proporción de hombres es de $86,3 \%$, mientras que la de mujeres es de $13,7 \%$.

En relación con la escolaridad, los datos muestran que los consumidores de coca tienen mayor nivel académico que los consumidores de crack. Por otro lado, en general los consumidores tienen una buena escolaridad, ya que el $62,8 \%$ tienen estudios por lo menos de bachillerato. Sabemos que el consumo de drogas tiene un efecto negativo en los estudios. Diversas investigaciones (Byrd, Weitzman y Doninger, 1996; Nappo, Galduróz y Noto, 1996) han demostrado que entre los factores asociados con el atraso escolar están el consumo de alcohol, cigarro y drogas ilícitas (entre ellas, la cocaína en todas sus presentaciones). 
En cuanto a la situación laboral de nuestra muestra, el $20,1 \%$ no estudia ni trabaja, y la proporción de estudiantes es muy baja (3,4\%); así, el $76,5 \%$ tiene un empleo, a pesar de que normalmente el consumo de cocaína, y sobre todo de crack, dificulta cualquier actividad que demande alguna responsabilidad. Este resultado es muy diferente a otros estudios. Por ejemplo, Ferreira et al. (2003) encontraron que en Brasil, la mitad de una muestra de consumidores de cocaína o crack se encontraba desempleada cuando se les hizo la entrevista. En nuestra muestra, la mayoría de los participantes ya tenían edad para dedicarse a algún trabajo formal. Además, había una proporción muy similar entre los consumidores de crack y los de cocaína. También este resultado es muy diferente al de otros estudios, ya que Dunn, Laranjeira, Silveira, Formigoni y Ferri (1996) encontraron que el $50 \%$ de los consumidores brasileños de cocaína que asistían a un tratamiento ambulatorio estaban desempleados.

Del total de pacientes, el 54,4\% ya habían recibido tratamiento de adicciones, de los cuales el $30,4 \%$ eran usuarios de cocaína y el $24 \%$, usuarios de crack. En ese sentido, también nuestro resultado es diferente a otros estudios. Por ejemplo, Ferreira et al. (2003) encontraron mayor número de internaciones entre los consumidores de crack que entre los consumidores de cocaína.

En nuestra población es muy cercana la proporción de consumidores de coca $(46 \%)$ con la de consumidores de crack (40,3\%). En México se ha encontrado que el consumo de crack ha aumentado en los últimos años (Secretaría de Salud, 2008); sin embargo, la proporción de consumidores de cocaína y crack es menos marcada en nuestro estudio $(5,7 \%)$, en contraste con los datos de la Quinta Encuesta Nacional de Adicciones (2008), que es del $1,78 \%$ en la población general.

Por otro lado, prácticamente ningún consumidor informó usar cocaína por vía intravenosa. Esto se debe probablemente a que realmente no consumían cocaína por esta vía, ya que en México se han informado muy pocos casos con este tipo de consumo. También es probable que los consumidores no quisieran revelar este dato, aunque creemos menos probable esta situación. O quizá los consumidores con esta vía de consumo asistan a otro tipo de centro de tratamiento, como Drogadictos Anónimos.

Respecto a los años de consumo, los consumidores de cocaína tienen más años de consumo que los consumidores de crack. De hecho, uno de los consumidores de cocaína informa haberla consumido por 23 años. Esta menor cantidad de años probablemente se deba a que el consumo de crack es mucho más reciente que el consumo de cocaína. En cuanto a la cantidad consumida por ocasión, hay una diferencia importante respecto al consumo de cocaína o crack, donde los consumidores de crack consumen cantidades más grandes de esta droga que los consumidores de cocaína.

Sobre los días de hospitalización, solo los consumidores de crack informaron haber estado internados más de 5 días; un usuario informa 12 días; otro, 21 días, y otro 30 días en el último año. Referente al tiempo invertido, no hay diferencias tan notables entre unos y otros consumidores.

Relativo al número de arrestos, en ambas muestras de consumidores ha habido este tipo de cuestiones legales. En cuanto a los consumidores de cocaína, el $8 \%$ de la muestra total han sido arrestados, mientras que de los consumidores de crack lo ha sido el $7,2 \%$. Por lo tanto, del $100 \%$ de consumidores, el $15,2 \%$ ha sido arrestado por causa de la droga. En cambio, en el estudio de Ferreira et al. (2003) encontraron que más del $57 \%$ de una población de consumidores de cocaína o crack habían estado presos a causa de la droga.

Una buena cantidad de usuarios consume alcohol $(n=81)$ concomitantemente con la cocaína o crack. Además, hay una gran proporción $(46,9 \%)$ que consume en alto riesgo, ya que consumen más de cuatro tragos estándar por ocasión de consumo. Esta proporción de usuarios es mayor que la de otros estudios. Ferreira et al. (2003) encontraron una proporción de 40,2\% de usuarios de cocaína o crack que consumían alcohol en cantidades de alto riesgo. Con los resultados obtenidos, es importante la prevención del consumo de alcohol por su asociación con el consumo de cocaína. 
Los consumidores informan que el alcohol puede ser el estímulo iniciador, o también puede ser uno de los desencadenantes de la búsqueda del efecto (Cañuelo y García, 2002).

El consumo del tabaco $(n=83)$ tiene una proporción muy parecida al alcohol, aunque es ligeramente más grande. En el estudio de Ferreira et al. (2003), también entre consumidores de cocaína o crack encontraron una proporción del $90 \%$.

Respecto a las otras drogas consumidas, en nuestros datos aparecen en primer lugar la marihuana, seguida de las anfetaminas y los inhalantes. La marihuana ha aparecido en todas las Encuestas Nacionales de Adicciones (1988, 1992, 1998, 2003 y 2008) en primer lugar. En la última ENA (Secretaría de Salud, 2008), los inhalables ocupan el tercer lugar de las drogas ilegales consumidas por la población general en nuestro país. Sin embargo, en nuestro estudio, las anfetaminas son más consumidas que los inhalantes, pero solo en el grupo de consumidores de cocaína, y los inhalantes quedarían en el tercer lugar solo en un consumidor, tanto de cocaína como de crack. Normalmente esta droga es consumida por menores de edad y nuestra muestra está constituida por mayores de edad. No se encontró un solo usuario que consumiera concomitantemente heroína.

Terracciano, Löckenhoff, Crum, Bienvenu y Costa (2008) señalan que los altos costos sociales e individuales asociados con el uso de drogas (entre ellas la cocaína) subraya la necesidad de estudiar los factores relacionados con tales conductas. Y las pequeñas diferencias entre las poblaciones pueden tener un efecto importante en las implicaciones clínicas para desarrollar tratamientos personalizados para las características de los consumidores de diferentes drogas, como la cocaína.

Con este tipo de investigaciones se puede conocer mejor a la población usuaria de este tipo de centros de adicciones, lo que contribuye en el conocimiento de las formas de prevención más eficientes para estas poblaciones, lo que podrá redundar en la disminución de los índices de incidencia de esta droga. La investigación sobre los correlatos de la adicción a las drogas da un insight para entender la etiología que apoya las po- líticas de prevención y los tratamientos de esta. Por ejemplo, podría haber controles toxicológicos similares al alcoholímetro como medio de disuasión, debido a la alta incidencia de accidentes de las personas intoxicadas por esta droga.

Sánchez, García y Nappo (2005) señalan que dos de los factores protectores más importantes para prevenir el consumo de cocaína entre una población de adolescentes en riesgo están el acceso a información sobre las sustancias y contar con una estructura familiar protectora (lazos afectivos entre padres e hijos, así como apoyo y respeto).

Hace falta hacer más investigación sobre los perfiles que tienen los usuarios de drogas en general, para así poder conocer sus características específicas, información importante para el desarrollo de tratamientos específicos para sustancias específicas.

Hay varias limitaciones por considerar cuando se interpretan los resultados. La primera es que la muestra no es representativa de la población, debido al tipo de muestreo que se efectuó. Por otro lado, algunos de los usuarios se negaron a dar información o no recordaban claramente su consumo anterior de drogas ilícitas.

\section{Referencias}

Byrd, R. S., Weitzman, M., \& Doninger, A. S. (1996). Increased drug use among old-for-grade adolescent. Archives of Pediatric \& Adolescent Medicine, 150, 470-476.

Cañuelo, B., \& García, M. del P. (2002). Perfil de los usuarios de Cocaína. Salud y Drogas, 2, 17-26.

Correia, D. S., Torres, A. A. P., Maia, E. M. C., de Sousa, F. F. L., \& Santos, V. J. C. (2010). Perfil de los usuarios de cocaína-crack en hospital de día. Memorias 11 congreso Virtual de Psiquiatría, I-8.

Duailibi, L.B., Ribeiro, M., \& Laranjeira, R. (2008). Profile of cocaine and crack users in Brazil. Cadernos de Saúde Pública, 24, 545-557. 
Dunn J., Laranjeira, R. R., Silveira, D. X., Formigoni, M. L. O. S., \& Ferri, C. P. (1996). Crack cocaína: an increase in use among patients attending clinics in Sao Paulo. Substance Use and Misuse, 31, 519-527.

Ferreira, O. F., Turchi, M. D., Laranjeira, R., \& Castelo, A. (2003). Perfil sociodemográfico e de padrões de uso entre dependentes de cocaína hospitalizados. Revista de Saude Publica, 37, 751-759.

Ferreira, P. E. M., \& Martini, R.K. (2001). Cocaína: lendas, história e abuso. Revista Brasileña de Psiquiatria, 23, 96-9.

Guimarães, C. F., Vieira dos Santos, D. V., Cavalari de Freitas, R., \& Araujo, R. B. (2008). Perfil do usuário de crack e fatores relacionados à criminalidade em unidade de internação para desintoxicação no Hospital Psiquiátrico São Pedro de Porto Alegre (RS). Revista de Psiquiatria do Rio Grande do Sul, 30, 101-108.

Herrero, M. J., Domingo-Salvany, A., Torrens, M., Brugal, M. T., \& Gutiérrez, F. (2008). Personality profile in young current regular users of cocaine. Substance Use \& Misuse, 43, 13781394.

Ladero, J. M., \& Martín, M. (2009). Cocaína: complicaciones orgánicas y psiquiátricas del consumo de cocaína. En P. Lorenzo, J. M. Ladero, J. C. Leza e I. Lizasoain (Eds.) Drogodependencias. Madrid: Médica Panamericana.

Nappo, S. A., Galduróz, J. C. F., \& Noto, A. R. (1996). Crack use in So Paulo. Substance Use and Misuse, 31, 565-579.

OPS. (2000). Guía internacional para vigilar el consumo del alcohol y sus consecuencias sanitarias. Ginebra: Organización Mundial de la Salud.

Oropeza, R. (2003). Desarrollo, aplicación y evaluación de un tratamiento breve para usuarios de cocaína (Tesis de doctorado. Facultad de Psicología, UNAM).
Oropeza, R., Medina-Mora, M. E., \& Sánchez-Sosa, J. J. (2007). Evaluación de un Tratamiento Breve para usuarios de cocaína. Revista Mexicana de Psicología, 24, 219-231.

Ribeiro, M., Dunn, J., Sesso, R., Dias, A. C., \& Laranjeira, R. (2006). Causes of death among crack cocaine users. Revista Brasileira de Psiquitria, 28, 196-202.

Sánchez, Z. M., \& Nappo, S. A. (2002). Seqüência de drogas consumidas por usuários de crack e fatores interferentes. Revista de Saúde Pública, 36, 420-430.

Sánchez, Z. M., Garcia, L., \& Nappo, S. A. (2005). Razões para o não-uso de drogas ilícitas entre jovens em situação de risco. Revista de Saúde Pública, 39, 599-605.

Secretaría de Salud (1988). Primera Encuesta Nacional de Adicciones. Secretaría de Salud, Consejo Nacional Contra las Adicciones. México: SS.

Secretaría de Salud (1992). Segunda Encuesta Nacional de Adicciones. Secretaría de Salud, Consejo Nacional Contra las Adicciones. México: SS.

Secretaría de Salud (1998). Tercera Encuesta Nacional de Adicciones. Secretaría de Salud, Consejo Nacional Contra las Adicciones. México: SS.

Secretaría de Salud (2003). Cuarta Encuesta Nacional de Adicciones. Secretaría de Salud, Consejo Nacional Contra las Adicciones. México: SS.

Secretaría de Salud (2008). Quinta Encuesta Nacional de Adicciones. Secretaría de Salud, Consejo Nacional Contra las Adicciones. México: SS.

Secretaría de Salud (2011). Encuesta Nacional de Adicciones 2011. Secretaría de Salud, Consejo Nacional Contra las Adicciones. México: SS. 
Terracciano, A., Löckenhoff, C., Crum, R. M., Bienvenu, O. J., \& Costa, P. T. (2008). Fivefactor model personality profiles of drug users. BMC Psychiatry, 8, 1-10.
Verdejo-García, A., \& Pérez-García, M. (2007). Profile of executive deficits in cocaine and heroin polysubstance users: common and differential effects on separate executive components. Psychopharmacology, 190, 517-530. 